\title{
Detection of Relevant Coronary Artery Disease Using Dual-Source Computed Tomography in a High Probability Patient Series
} _ Comparison With Invasive Angiography

\author{
Johannes Rixe, MD; Andreas Rolf, MD; Guido Conradi, MD; Helge Moellmann, MD; \\ Holger Nef, MD; Thomas Neumann, MD; Holger Steiger, MD; \\ Christian W. Hamm, MD, FESC, FACC; Thorsten Dill, MD, FESC
}

\begin{abstract}
Background Computed tomography (CT) enables detection of coronary artery stenoses, but its use is limited by deficient evaluation at elevated heart rates. The accuracy of dual-source CT (DSCT) for the detection of coronary artery disease (CAD) was assessed in 76 patients at high probability of CAD without heart rate control and compared with quantitative coronary angiography (QCA).

Methods and Results The 76 patients (47 males, mean age $65.5 \pm 10$ years) underwent DSCT without preceding heart rate control. Data sets were evaluated by 2 observers in consensus with respect to stenoses $>50 \%$ decreased diameter. QCA served as the standard of reference. Mean heart rate during scanning was $68 \pm 9$ beats per min, and the average Agatston score was 337 \pm 560 . Of 1,160 coronary artery segments, all but 3 were visualized artefactfree; 58 coronary stenoses were correctly detected by CT angiography. In the segment-based analysis, sensitivity was $98.3 \%$, specificity $99.2 \%$ and accuracy $99 \%$; patient based analysis revealed a sensitivity of $100 \%$, specificity of $83.3 \%$ and overall accuracy of $92.1 \%$.

Conclusions Even at elevated heart rates, DSCT can reliably detect coronary artery stenoses and the results correlate well with those for invasive coronary angiography. (Circ J 2009; 73: 316-322)
\end{abstract}

Key Words: Cardiac imaging; Coronary angiography; Coronary artery disease; Dual source computed tomography; Heart rate control

B ecause noninvasive visualization of coronary arteries and detection of relevant coronary artery stenoses using multidetector computed tomography (MDCT) have so far been challenging because of the small dimensions of the coronary arteries and their rapid motion, the improvement of temporal, as well as spatial, resolution has been the cardinal aims of scanner development over the past decade. However, coronary CT angiography (CTA) using 16-slice MDCT is still affected by numerous limitations, mostly partial volume effects caused by coronary calcification and motion artefacts because of higher coronary velocities combined with limited temporal resolution at heart rates $>65$ beats per min (bpm) $)^{1,2}$ Even visualization of coronary arteries using 64-slice MDCT with an increased temporal resolution of $165 \mathrm{~ms}$ is limited by severe coronary calcification and motion artefacts at heart rates $>65 \mathrm{bpm}$, resulting in up to $12 \%$ of coronary segments being unsuitable for quantitative evaluation? ${ }^{-5}$

To overcome motion artefacts at higher heart rates, dual

(Received June 8, 2008; revised manuscript received October 1, 2008; accepted October 9, 2008; released online December 27, 2008)

Department of Cardiology, Kerckhoff Heart Centre, Bad Nauheim, Germany

Mailing address: Johannes Rixe, MD, Kerckhoff Heart Centre, Department of Cardiology/Cardiovascular Imaging, Benekestrasse 2-8, D-61231 Bad Nauheim, Germany. E-mail: j.rixe@kerckhoff-klinik. de

All rights are reserved to the Japanese Circulation Society. For permissions, please e-mail: cj@j-circ.or.jp
source-CT (DSCT) technology has recently been introduced. By using $2 \mathrm{X}$-ray tubes mounted onto a single gantry at an angle of $90^{\circ}$, a duplication of temporal resolution from $165 \mathrm{~ms}$ to $83 \mathrm{~ms}$ is provided with a mono-segment reconstruction mode6 Recent studies using DSCT have shown encouraging results for visualization of the coronary arteries independent of heart rate; however, only small numbers of patients have been included and the correlation to quantitative coronary angiography (QCA) has so far barely been provided? ${ }^{70}$

Thus, the purpose of the present study was to assess the feasibility of DSCT for detection of relevant coronary artery stenoses in a cohort of 76 patients with clinically suspected coronary artery disease $(\mathrm{CAD})$ and compare the results with those from invasive angiography without preceding heart rate control.

\section{Methods}

\section{Patients}

During a 2-month period, 76 consecutive patients (47 males, all Caucasians; mean age, $65.5 \pm 10$ years) referred for invasive coronary angiography (CAG) because of suspected CAD were included in our study. Clinical signs of CAD were typical chest pain in 50 patients $(65.8 \%)$, positive stress testing in $15(19.7 \%)$ and both indicators in 11 patients $(14.5 \%)$. As it had been identically performed in previous studies, a positive stress test was not mandatory for inclusion in our study, but all patients with clinical 
symptoms as the exclusive criterion for inclusion reported typical chest pain suspicious of CAD2,11 Patient characteristics are shown in Table 1. Only patients in a stable clinical condition and without contraindications for the administration of iodinated contrast agents were investigated. Patients with coronary bypass grafts, prior stent implantation, valve prostheses and cardiac pacemakers were excluded, as were patients with atrial fibrillation, because the aim of the study was not to evaluate image quality in patients with atrial fibrillation. Written informed consent was given by all individuals included in our study, and the study was approved by the institutional review board.

\section{DSCT Protocol}

DSCT was performed with a SOMATOM Definition CT scanner (Siemens Medical Solutions, Forchheim, Germany) without heart rate modulation by oral or intravenous administration of $\beta$-blockers prior to the scanning procedure, but 45 patients $(59.2 \%)$ were under continuous $\beta$-blocker medication and those patients had an average heart rate of $63.6 \pm 7.9 \mathrm{bpm}$. Overall, 36 patients had a heart rate $>65 \mathrm{bpm}$ during scanning (47.4\% of all patients), of whom 24 had a heart rate $>70 \mathrm{bpm}$ ( $31.6 \%$ of all patients included).

After placing an i.v. line in an antecubital vein, and connecting the electrocardiogram (ECG) electrodes in the standard positions, all patients received $0.8 \mathrm{mg}$ of isosorbide dinitrate sublingually immediately before scanning. In agreement with previously described protocols, a test bolus approach was performed, determining the contrast agent transit time $(10 \mathrm{ml}$ of iopamidole contrast, containing $370 \mathrm{mg}$ iodine $/ \mathrm{ml}$, followed by $50 \mathrm{ml}$ of isotonic saline, both at $5 \mathrm{ml} / \mathrm{s}$ ) by calculating the time between beginning the contrast agent injection and maximum enhancement in the ascending aorta! ${ }^{12,13}$ For coronary artery scanning, the individual delay was calculated and $60 \mathrm{ml}$ of contrast agent were injected at $5 \mathrm{ml} / \mathrm{s}$, followed by $50 \mathrm{ml}$ of saline. Data acquisition was performed from the level of the tracheal bifurcation to the diaphragm in the craniocaudal direction using a detector collimation of $32 \times 0.6 \mathrm{~mm}$, slice acquisition $64 \times 0.6 \mathrm{~mm}$ by means of a z-flying focal spot ${ }^{14}$ Gantry rotation time was $330 \mathrm{~ms}$; pitch $0.2-0.43$, adapted manually according to heart rate. Tube current was $360 \mathrm{~mA}$, and tube voltage was $120 \mathrm{kV}$ for both tubes. In accordance with previously published studies for dose reduction algorithms, ${ }^{15-17}$ ECG-gated tube current modulation and automatic radiation exposure control were both used in all patients, and the pulsing window was fixed from $65 \%$ to $70 \%$ of the RRcycle with heart rates $<70 \mathrm{bpm}$, or from $40 \%$ to $70 \%$ of the RR-cycle with heart rates $>70 \mathrm{bpm}$.

\section{Image Reconstruction}

Retrospective ECG-gated image reconstruction was performed with a half-scan and single RR-interval reconstruction algorithm, providing for a temporal resolution of $83 \mathrm{~ms}$, as described earlier6 The first image reconstruction was performed at $70 \%$ of the RR-cycle, followed by an automatically generated reconstruction using dedicated reconstruction software, which automatically generates the most quiescent phase during the RR-cycle by calculating a motion strength function between several reconstructions at low resolution over the cardiac cycle and identifying periods of low difference between neighboring phases (BestDiast/ BestSyst $^{\circledR}$, Siemens Medical Solutions) ${ }^{18}$ If motion artefacts were present, additional reconstructions in 5\% increments and decrements were rendered, as described5,19-22
Table 1 Clinical Characteristics of the Enrolled Patients

\begin{tabular}{lc}
\hline \hline No. of patients & 76 \\
\hline No. of patients with $H R>65 />70$ bpm & $36 / 24$ \\
Mean heart rate $($ bpm $)$ & $68 \pm 9($ range $49-85)$ \\
Mean Agatston score & $100 \pm 560($ range $0-2,650)$ \\
Male gender & $47(62 \%)$ \\
Mean age (years) & $65 \pm 10$ \\
Diabetes mellitus & $21(28 \%)$ \\
Arterial hypertension & $64(84 \%)$ \\
Hypercholesterolemia & $45(59 \%)$ \\
Family history of CAD & $21(28 \%)$ \\
Smoking & $9(12 \%)$ \\
Obesity & $33(43 \%)$ \\
Mean weight $(\mathrm{kg}) / \mathrm{BMI}$ & $84.5 \pm 17.6 / 28.7 \pm 4.8$ \\
Mean height $(\mathrm{m})$ & $1.75 \pm 0.1$ \\
\hline
\end{tabular}

$H R$, heart rate; bpm, beats per min; $C A D$, coronary artery disease; $B M I$, body mass index.

For image reconstruction, a slice thickness of $0.6 \mathrm{~mm}$ and an increment of $0.3 \mathrm{~mm}$ were chosen. As performed by Flohr et al, reconstructions were rendered by using a medium-sharp (B26F) convolution kernel14 In the presence of calcified plaques, a sharp convolution kernel (B46F) was additionally reconstructed and used for evaluation.

\section{Data Analysis}

For postprocessing, the DSCT data sets with highest image quality were transferred to an offline workstation (LEONARDO ${ }^{\circledR}$, Siemens Medical Solutions) and evaluated by 2 experienced investigators in consensus blinded to each patient's identity and clinical history.

Coronary segments were defined using the American Heart Association (AHA)/American College of Cardiology (ACC) 16-segment model23 All segments were measured for diameter, and all segments $<1.5 \mathrm{~mm}$ in diameter were excluded from the analysis. Segments were classified as "evaluable" or "unevaluable", and assessed for the presence of stenoses $>50 \%$ lumen reduction, as well as for the presence of occlusions. Depending on vessel morphology and image quality, various postprocessing techniques were used in the evaluation. After assessment of axial and oblique multiplanar reconstructions (MPR), oblique maximum intensity projections, curved multiplanar reconstructions (curved MPR), and 3-dimensional volume-rendering technique projections (VRT) were performed.

$Q C A$

Selective conventional CAG served as the standard of reference and was performed $24-48 \mathrm{~h}$ after the DSCT procedure using standard techniques by a skilled observer who was unaware of the results obtained by DSCT. Quantitative evaluation was performed on an offline workstation with dedicated software (QuantCor. QCA ${ }^{\circledR}$; PieMedical Imaging, Maastricht, The Netherlands) using the AHA/ACC 16-segment coronary model. Coronary segments with a diameter $<1.5 \mathrm{~mm}$ were excluded from analysis, and a reduction $>50 \%$ of the luminal diameter compared with the proximal reference diameter was considered a significant stenosis.

\section{Statistical Analysis}

Quantitative variables are described as mean \pm standard deviation, and categorical variables are presented as counts and percentages. Diagnostic accuracy for detection of relevant coronary lesions by DSCT was expressed as sensitivi- 

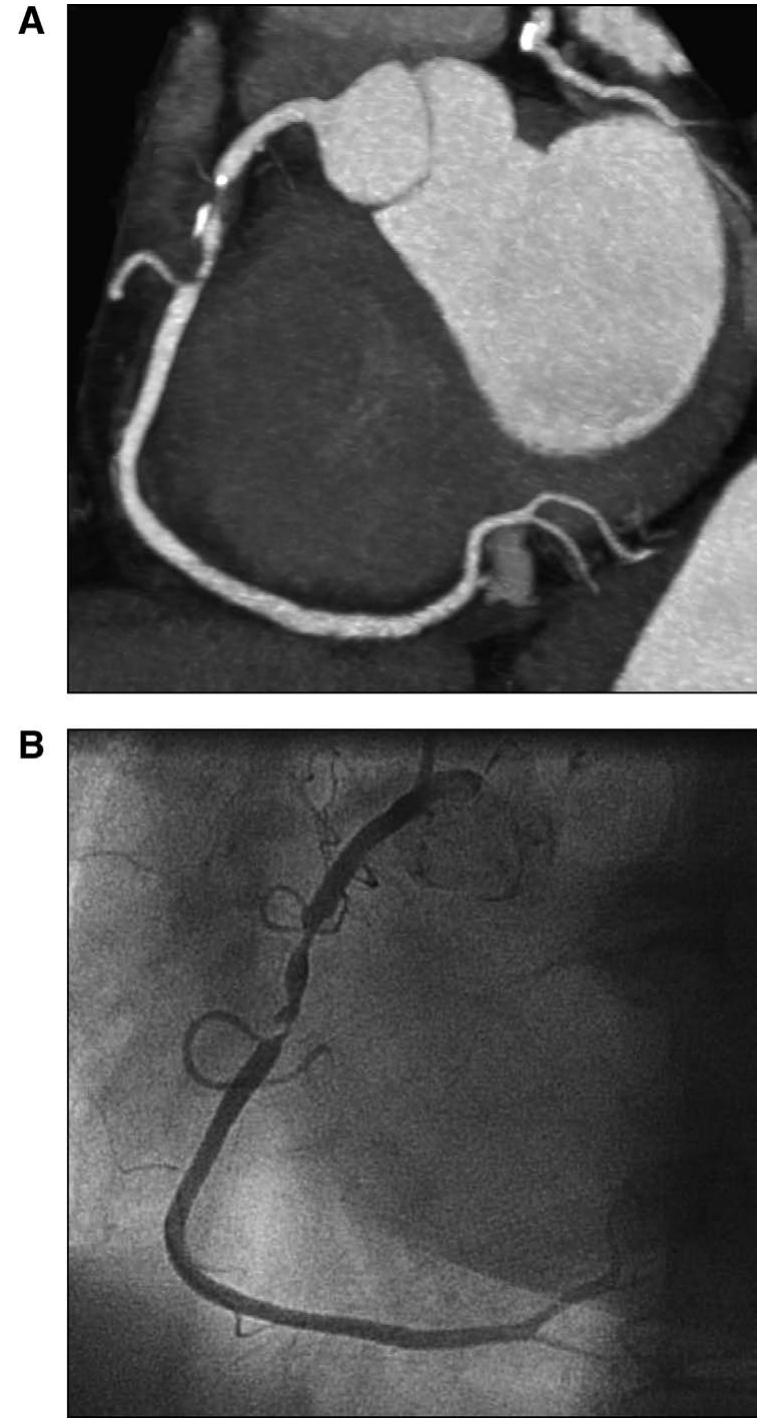

Fig 1. Maximum intensity projection of the right coronary artery (A) demonstrates a significant stenosis in the proximal portion. Reconstructed slice thickness is $5 \mathrm{~mm}$, and heart rate is 65 beats per min The time instant of image reconstruction was $71 \%$ of the RR-cycle. The patient was referred for conventional angiography and the stenosis was confirmed $(\mathbf{B})$.

ty, specificity, positive predictive value (PPV) and negative predictive value (NPV), calculated from the chi-square test of contingency.

Calculations were performed for coronary segments, coronary arteries and in patient-based analysis. If at least 1 coronary segment was unevaluable on DSCT, it was regarded as having a relevant stenosis because of the clinical consequence of invasive angiography if the presence of a stenosis could not be ruled out.

\section{Results}

DSCT was performed successfully and without complications in all 76 patients enrolled; the mean scan and breathhold time for CTA scan was $9.68 \pm 1.73 \mathrm{~s}$. Mean heart rate during scanning was $68 \pm 9 \mathrm{bpm}$ (range $49-85 \mathrm{bpm}$ ) and the average Agatston score was $337 \pm 560$ (range 0-2,650).

\section{Assessment of Image Quality}

A total of 1,080 coronary segments and 304 coronary arteries were included in our study, of which 1,072 coronary segments $(99.3 \%)$ and 296 coronary arteries $(97.4 \%)$ could be assessed without artefacts; 80 coronary segments with a diameter $<1.5 \mathrm{~mm}$ were excluded from analysis, but no coronary segment impaired by artefacts because of motion or blurring was excluded. Three patients $3.9 \%$ of all patients enrolled, $0.28 \%$ of all coronary segments) had CT angiograms severely affected by motion artefacts, of which all were because of premature beats of supraventricular $(n=2)$ or ventricular origin $(n=1)$. No coronary segment was considered unevaluable because of motion artefacts because of elevated heart rates. In 5 patients $(6.6 \%$ of all patients) with an average Agatston score of $988 \pm 1,010,5$ coronary segments $(0.5 \%$ of all segments included) severe coronary calcifications were present, significantly impairing image quality by obliterating the coronary lumen. These 5 segments were regarded as having significant stenosis.

Under exclusion of the automated reconstruction tool described in the Methods, the overall number of different time instants necessary for each data set to obtain diagnostic image quality was $1 \pm 0.3$.

In our patient series, optimal image quality could be achieved using exclusively diastolic reconstructions at $65 \%$ or $70 \%$ of the RR-cycle in 55 patients (72\% of all patients). These data sets were acquired at a mean heart rate of $65 \pm$ $4 \mathrm{bpm}$, which was significantly lower than the mean heart rate of patients whose raw data were ideally reconstructed at systolic time instants of the cardiac cycle (the corresponding mean heart rate was $76 \pm 5.3 \mathrm{bpm}, \mathrm{P}<0.05)$. The optimal time instants for systolic reconstructions were $35 \%$ and $40 \%$, respectively, with a total of 21 data sets ( $28 \%$ of all patients). In a subanalysis of patients with heart rate $>65 \mathrm{bpm}(\mathrm{n}=36)$, a systolic reconstruction of raw data proved satisfactory only for patients with heart rate $>70 \mathrm{bpm}$, as all data sets with best image quality at $35 \%$ or $40 \%$ were obtained in patients with a heart rate $>70 \mathrm{bpm}$.

\section{Comparison of DSCT and QCA}

QCA demonstrated CAD in 40 patients (prevalence in total patients, $53 \%$ ). Among these 40 patients, 59 coronary stenoses $>50 \%$ diameter reduction were detected by QCA ( 2 in left main, 29 in left anterior descending, 15 in left circumflex and 13 in right coronary artery; Fig 1).

Of the 1,072 coronary segments classified as evaluable on DSCT, 58 showed significant stenoses. One stenotic lesion of coronary segment 3 was found to be $>50 \%$ reduced on QCA but was missed by DSCT. Nine coronary segments were classified as false-positive by DSCT: 8 were unevaluable and thus estimated as having significant stenosis, but did not show stenosis on QCA. One further segment was evaluated as false-positive because the degree of lumen reduction was overestimated; the remaining 1,012 coronary segments were assessed as true negative on DSCT (Fig 2).

Thus, on a per-segment analysis, DSCT revealed a sensitivity of $98.3 \%$, specificity of $99.1 \%$, and the NPV and PPV were $99.9 \%$ and $86.6 \%$, respectively. Vessel-based and patient-based analyses revealed a sensitivity of $98.3 \%$ (patient-based $100 \%$ ) and specificity of $97.1 \%$ (patientbased $83.3 \%$ ). The NPV and PPV values were $99.6 \%$ and $89.4 \%$ in the vessel-based and $100 \%$ and $86.9 \%$ in the perpatient analyses, respectively (Table 2).

Overall diagnostic accuracy was $99.1 \%$ in the coronarysegment-based analysis; vessel- and patient-based analyses 
A
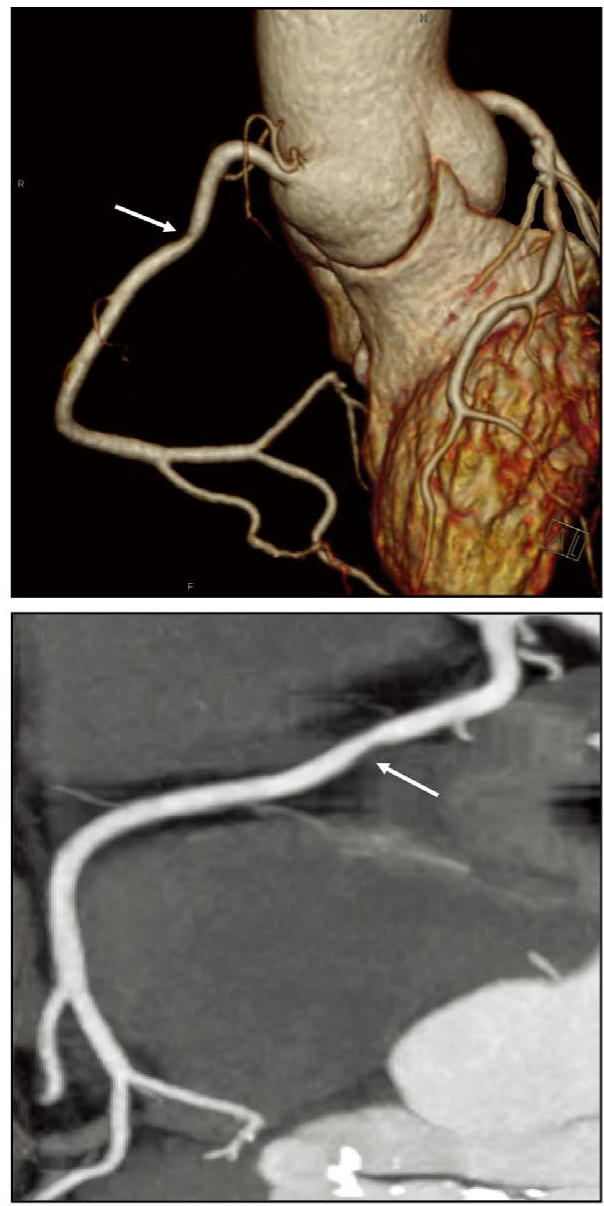

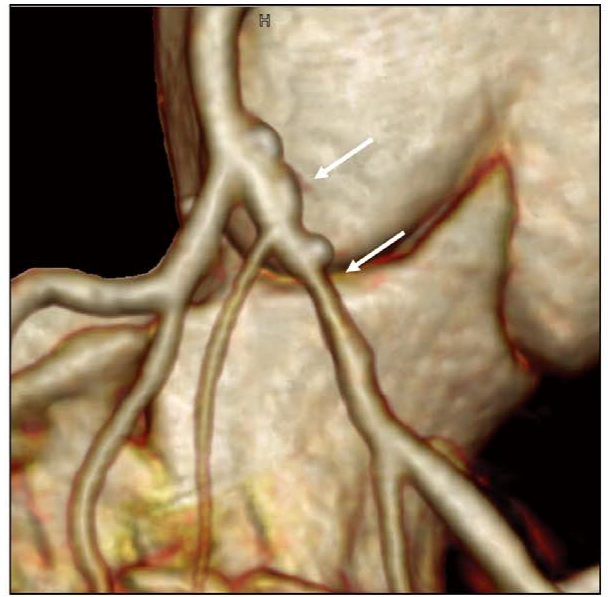

a
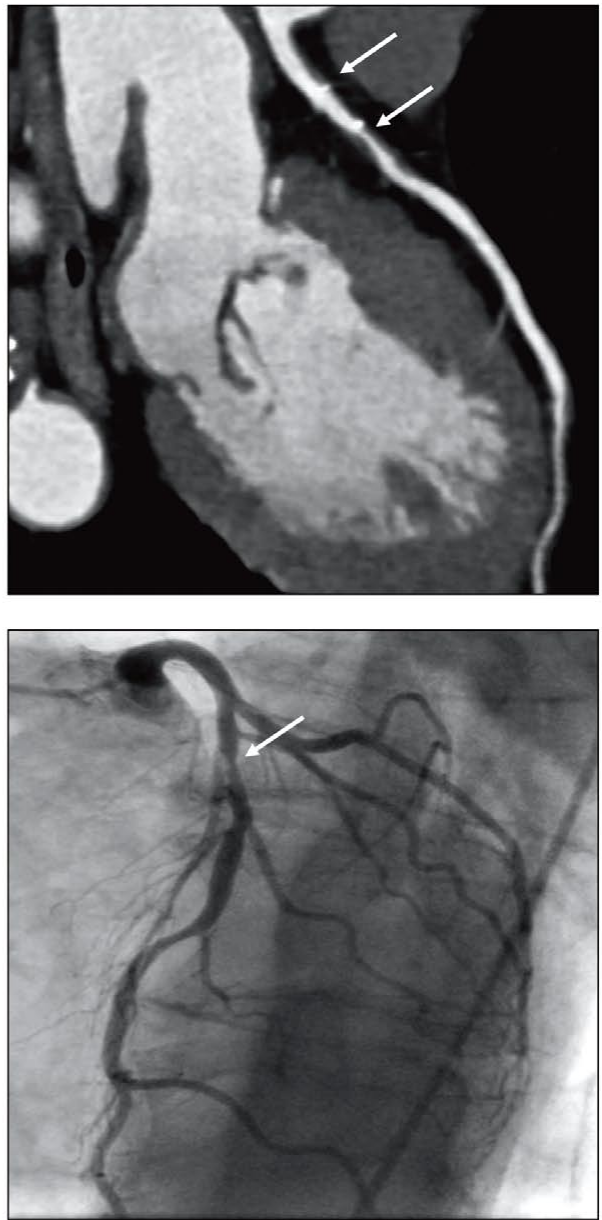

B

C

D

Fig 2. Acknowledging that volume-rendered projection (VRT) images are not suitable for exclusion or even detection of relevant coronary artery disease, the VRT and curved multiplanar reconstruction (cMPR) of the right coronary artery (A) show noncalcified plaque (arrows), but no findings suspicious of relevant stenoses, whereas an intermediate stenosis is present on the VRT image of the proximal left anterior descending artery $(\mathbf{B}$, arrows). The cMPR $(\mathbf{C})$ with a reconstructed slice thickness of $0.75 \mathrm{~mm}$ shows a stenosis of intermediate severity and partly calcified plaque with signs of positive remodeling (arrows). Heart rate during scanning was 62 beats per min, and the time instant for image reconstruction was $70 \%$ of the cardiac cycle. Conventional angiography confirmed these findings (D, arrow).

revealed an overall accuracy of $97 \%$ and $92.1 \%$, respectively.

Regarding patients with elevated heart rates, sub analyses for patients with $>65 \mathrm{bpm}$ or $>70 \mathrm{bpm}$ revealed a sensitivity, specificity, PPV and NPV that were not substantially different to the statistical parameters of the patient series in general. At least, sensitivity and NPV values remained remarkably constant, whereas sensitivity ranged from $98.3 \%$ in the segment- and coronary-based calculations to $100 \%$ in all patient-based analyses, and the NPV ranged from $99.6 \%$ 
Table 2 Comparison of DSCT and QCA

\begin{tabular}{lccccc}
\hline \hline Type of analysis & Evaluable & Sensitivity & Specificity & $P P V$ & $N P V$ \\
\hline Segment-based & $1,072(99.3 \%)$ & $98.3 \%$ & $99.1 \%$ & $86.6 \%$ & $99.9 \%$ \\
95\%CI & & $89.7-99.9$ & $98.3-99.6$ & $75.5-93.3$ & $99.4-100$ \\
Coronary-based & $296(97.4 \%)$ & $98.3 \%$ & $97.1 \%$ & $89.4 \%$ & $99.6 \%$ \\
$95 \%$ CI & & $89.9-99.9$ & $93.9-98.5$ & $78.8-95.3$ & $97.3-100$ \\
Patient-based & $68(89.5 \%)$ & $100 \%$ & $83.3 \%$ & $86.9 \%$ & $100 \%$ \\
95\%CI & & $89.1-100.0$ & $66.5-93.0$ & $73.0-94.6$ & $85.9-100$ \\
Patients with HR>65 bpm & $34(94.4 \%)$ & $100 \%$ & $88.2 \%$ & $90.5 \%$ & $100 \%$ \\
$95 \%$ CI & & $79.0-100.0$ & $62.3-97.9$ & $68.2-98.3$ & $74.7-100$ \\
Patients with HR $>70 \mathrm{bpm}$ & $22(91.7 \%)$ & $100 \%$ & $85.7 \%$ & $83.3 \%$ & $100 \%$ \\
$95 \%$ CI & & $65.5-100.0$ & $56.2-97.5$ & $50.1-97.1$ & $69.9-100$ \\
\hline
\end{tabular}

In segment-based, vessel-based and even patient-based analyses, individuals with at least 1 coronary segment considered unevaluable on DSCT were regarded as having arelevant coronary stenosis.

DSCT, dual-source computed tomography; QCA, quantitative coronary angiography; PPV, positive predictive value; NPV, negative predictive value; CI, confidence interval. Other abbreviation see in Table 1.

in the coronary-based calculation to $100 \%$ in all patientbased analyses.

\section{Radiation Dose}

Mean effective radiation dose for all patients was $13.8 \pm 6.7 \mathrm{mSv}$; for patients with a heart rate $>70 \mathrm{bpm}(\mathrm{n}=36)$, it was $14.3 \pm 5.6 \mathrm{mSv}$.

\section{Discussion}

Our study demonstrates that the improvement in temporal resolution for coronary artery imaging by $\mathrm{CT}$ enables accurate detection of relevant coronary artery stenoses, even at elevated heart rates, and thus broadens the spectrum of patients that can be assessed non-invasively.

CAG using 16- and 64-slice MDCT has become an increasingly reliable tool for visualizing the coronary arteries and has proved particularly reliable for ruling out of CAD in selected patient cohorts. The high NPV for ruling out CAD has prompted the current European Society of Cardiology (ESC) guidelines on management of stable angina to recommend MDCT for patients with a low pretest probability of CAD and inconclusive stress testing? 4 Additionally, recently published studies underline the feasibility of coronary CTA to even detect coronary plaque and therefore clinically silent $\mathrm{CAD}^{25-27} \mathrm{~A}$ certain prognostic impact on the detection of coronary calcification and non-obstructive $\mathrm{CAD}$ is therefore assumed ${ }^{28-30}$

However, in clinical practice 2 major limitations are apparent: Firstly, coronary calcifications lead to overestimation of coronary stenoses because of blurring artefacts and total obstruction of the coronary lumen, and may significantly impair diagnostic accuracy ${ }^{31,32}$ and thus lead to unnecessary invasive angiography. Secondly, the occurrence of motion artefacts at elevated heart rates is a common problem, which most frequently affects the right coronary artery because of its extensive motion during the cardiac cycle. A low heart rate during scanning has therefore been an essential requirement to obtain a data set suitable for quantitative analysis, ${ }^{32-34}$ which so far has required a consistent heart rate lowering protocol using oral or intravenous administration of $\beta$-blockers!-4,13,35-38 Hence, the mean heart rate during scanning in studies using 64-slice MDCT is considerably below $65 \mathrm{bpm}, 5$

To overcome the occurrence of motion artefacts at higher heart rates, 2 technical approaches have been developed; multi-segment reconstruction algorithms combine data sets from 2 or even more cardiac cycles for reconstruction, achieving improved temporal resolution, but they disregard the inter-heartbeat variability of coronary artery position and require low table pitches 39,40

By providing a temporal resolution of $83 \mathrm{~ms}$ in a monosegment reconstruction mode, DSCT enables motion artefact-free visualization of coronary arteries, independent of heart rate, and therefore a significant decrease of motion artefacts in clinical practice, 6

In this prospective study we assessed the feasibility of new DSCT technology to detect coronary artery stenoses in a cohort of 76 consecutive patients without precedent heart rate lowering medication. An overall diagnostic accuracy of $99.1 \%$ and excellent NPV of $99.9 \%$ in segment-based analysis was achieved without excluding any coronary segments from statistical analysis because of artefacts. Only $0.7 \%$ of all coronary segments were unevaluable on DSCT, and thus regarded as having significant stenosis, but none of these was assessed unevaluable because of motion artefacts caused by elevated heart rate.

There are only a few published studies evaluating the feasibility of DSCT for the identification of relevant coronary artery stenoses in patients, which differs significantly from our study of patients with a relatively high pre-test probability for CAD?,10,11 Considering the study by Busch et al, DSCT presents itself superior to 64-slice MDCT for the detection of CAD: in a small cohort of 10 patients each, DSCT was superior to 64-slice MDCT for the detection of CAD; DSCT demonstrated a sensitivity and specificity of $100 \%$ for both, whereas thea 64-slice scanner achieved a sensitivity of $100 \%$, but a reduced specificity of $90 \%$; the mean heart rate of patients examined with the DSCT scanner was $80 \mathrm{bpm}$, but the mean heart rate of patients scanned by MDCT was not given?

Similar to the results of our study, Leber et al, Scheffel et al, and Ropers et al demonstrated, that an excellent overall diagnostic accuracy and an NPV of 99\% in per segment analysis can be achieved using DSCT for the detection of CAD in their studies with 90, 30, and 100 enrolled patients, respectively! $10,11,41$ The patients enrolled in the studies of Scheffel et al and Leber et al had mean heart rates of 73 and $70.3 \mathrm{bpm}$, respectively, and a pre-test likelihood of CAD ranging from "low" to "intermediate" in the study by Leber et al to "high" in the study by Scheffel et al. In the study by Ropers et al, the mean heart rate was $64 \pm 13 \mathrm{bpm}$, which is in fact at the level of earlier studies with 64-slice MDCT 41 In addition, the prevalence of CAD in the latter study is 
lower than in all studies of DSCT previously published (only $41 \%$ ). The potential of DSCT technology is better tested in a cohort of patients with a higher prevalence of CAD (eg, $53 \%$ in our study), while acknowledging that patients with a high pre-test likelihood for CAD do not represent the patient cogroup recommended for coronary MDCT by current Eurpean guidelines 24

The recently published study by Husmann et al also included patients with a high probability for CAD, but in fact there were only 25 patients in the high-risk subgroup, and furthermore, the authors describe an improved PPV for the diagnosis of relevant CAD in that subgroup. ${ }^{42}$ Taking into account that patients with a high probability for CAD tend to have higher amounts of calcification, this conclusion appears to be relatively implausible.

\section{Study Limitations}

A major limitation is that the mean heart rate during scanning was $68 \pm 9 \mathrm{bpm}$, which is not considerably higher than that reported in studies using 64-slice MDCT ${ }^{4,19,35}$ However, in comparison with those studies, our data suggest a considerably increased diagnostic accuracy, which might be at least partially related to optimized image quality at elevated heart rates. Another limitation is that we did not enrol patients with coronary stents or atrial fibrillation, both clinically relevant conditions that should be the subject of further studies.

In conclusion, this study demonstrates that noninvasive CAG using CT has become a reliable alternative to invasive diagnostic procedures. The present patient cohort tested with a high prevalence of $53 \%$ for CAD was very well suited to testing the accuracy of the new DSCT technique, while acknowledging that it is not the patient group that is aimed for investigation with noninvasive $\mathrm{CTA}^{24}$ This study revealed a high accuracy for the detection of relevant coronary artery stenosis, even in a patient series of high pre-test probability for CAD, and confirmed the high NPV in patients with higher heart rates, and thus using DSCT may avoid the unnecessary invasive procedures related to inconclusive findings on CTA by previous scanner generations.

\section{Disclosure}

None.

\section{References}

1. Mollet NR, Cademartiri F, Nieman K, Saia F, Lemos PA, McFadden $\mathrm{EP}$, et al. Multislice spiral computed tomography coronary angiography in patients with stable angina pectoris. J Am Coll Cardiol 2004; 43: $2265-2270$

2. Ropers D, Baum U, Pohle K, Anders K, Ulzheimer S, Ohnesorge B, et al. Detection of coronary artery stenoses with thin-slice multi-detector row spiral computed tomography and multiplanar reconstruction. Circulation 2003; 107: 664-666.

3. Nikolaou K, Knez A, Rist C, Wintersperger BJ, Leber A, Johnson T, et al. Accuracy of 64-MDCT in the diagnosis of ischemic heart disease. Am J Roentgenol 2006; 187: 111-117.

4. Raff GL, Gallagher MJ, O'Neill WW, Goldstein JA. Diagnostic accuracy of noninvasive coronary angiography using 64-slice spiral computed tomography. J Am Coll Cardiol 2005; 46: 552-557.

5. Ropers D, Rixe J, Anders K, Kuttner A, Baum U, Bautz W, et al. Usefulness of multidetector row spiral computed tomography with $64 \times 0.6-\mathrm{mm}$ collimation and $330-\mathrm{ms}$ rotation for the noninvasive detection of significant coronary artery stenoses. Am J Cardiol 2006; 97: $343-348$.

6. Flohr TG, McCollough CH, Bruder H, Petersilka M, Gruber K, Suss $\mathrm{C}$, et al. First performance evaluation of a dual-source CT (DSCT) system. Eur Radiol 2006; 16: 256-268.

7. Achenbach S, Ropers D, Kuettner A, Flohr T, Ohnesorge B, Bruder $\mathrm{H}$, et al. Contrast-enhanced coronary artery visualization by dualsource computed tomography: Initial experience. Eur J Radiol 2006;
57: $331-335$.

8. Busch S, Nikolaou K, Johnson T, Rist C, Knez A, Reiser M, et al. Quantification of coronary artery stenoses: Comparison of 64-slice and dual source $\mathrm{CT}$ angiography with cardiac catheterization. Radiologe 2007; 47: 295-300 (in German).

9. Johnson TR, Nikolaou K, Wintersperger BJ, Leber AW, von Ziegler $\mathrm{F}$, Rist C, et al. Dual-source CT cardiac imaging: Initial experience. Eur Radiol 2006; 16: 1409-1415.

10. Scheffel H, Alkadhi H, Plass A, Vachenauer R, Desbiolles L, Gaemperli O, et al. Accuracy of dual-source CT coronary angiography: First experience in a high pre-test probability population without heart rate control Eur Radiol 2006; 16: 2739-2747.

11. Leber AW, Johnson T, Becker A, von Ziegler F, Tittus J, Nikolaou $\mathrm{K}$, et al. Diagnostic accuracy of dual-source multi-slice CT-coronary angiography in patients with an intermediate pretest likelihood for coronary artery disease. Eur Heart J 2007; 28: 2354-2360.

12. Achenbach S, Ulzheimer S, Baum U, Kachelriess M, Ropers D, Giesler T, et al. Noninvasive coronary angiography by retrospectively ECG-gated multislice spiral CT. Circulation 2000; 102: 2823-2828.

13. Ropers D, Pohle FK, Kuettner A, Pflederer T, Anders K, Daniel WG, et al. Diagnostic accuracy of noninvasive coronary angiography in patients after bypass surgery using 64-slice spiral computed tomography with 330-ms gantry rotation. Circulation 2006; 114: $2334-$ 2341; quiz 2334.

14. Flohr T, Stierstorfer K, Raupach R, Ulzheimer S, Bruder H. Performance evaluation of a 64-slice CT system with z-flying focal spot. Rofo 2004; 176: $1803-1810$.

15. Hausleiter J, Meyer T, Hadamitzky M, Huber E, Zankl M, Martinoff $\mathrm{S}$, et al. Radiation dose estimates from cardiac multislice computed tomography in daily practice: Impact of different scanning protocols on effective dose estimates. Circulation 2006; 113: 1305-1310.

16. Deetjen AG, Möllmann S, Conradi G, Rolf A, Schmermund A, Hamm CW, et al. Use of Automatic exposure control in multislice computed tomography of the coronaries: Comparison of 16-slice and 64-slice scanner data with conventional coronary angiography. Heart 2007; 93: 1040-1043.

17. Dill T, Deetjen A, Ekinci O, Mollmann S, Conradi G, Kluge A, et al. Radiation dose exposure in multislice computed tomography of the coronaries in comparison with conventional coronary angiography. Int J Cardiol 2008; 124: 307-311.

18. Hoffmann MH, Lessick J, Manzke R, Schmid FT, Gershin E, Boll DT, et al. Automatic determination of minimal cardiac motion phases for computed tomography imaging: Initial experience. Eur Radiol 2006; 16: $365-373$.

19. Leschka S, Alkadhi H, Plass A, Desbiolles L, Grunenfelder J, Marincek B, et al. Accuracy of MSCT coronary angiography with 64-slice technology: First experience. Eur Heart J 2005; 26: $1482-$ 1487.

20. Leschka S, Husmann L, Desbiolles LM, Gaemperli O, Schepis T, Koepfli $\mathrm{P}$, et al. Optimal image reconstruction intervals for non-invasive coronary angiography with 64-slice CT. Eur Radiol 2006; 16: $1964-1972$.

21. Matsutani H, Sano T, Kondo T, Morita H, Arai T, Sekine T, et al. ECG-edit function in multidetector-row computed tomography coronary arteriography for patients with arrhythmias. Circ J 2008; 72: 1071-1078.

22. Nagatani Y, Takahashi M, Takazakura R, Nitta N, Murata K, Ushio $\mathrm{N}$, et al. Multidetector-row computed tomography coronary angiography: Optimization of image reconstruction phase according to the heart rate. Circ J 2007; 71: 112-121.

23. Austen WG, Edwards JE, Frye RL, Gensini GG, Gott VL, Griffith LS, et al. A reporting system on patients evaluated for coronary artery disease: Report of the Ad Hoc Committee for Grading of Coronary Artery Disease, Council on Cardiovascular Surgery, American Heart Association. Circulation 1975; 51(Suppl): 5-40.

24. Fox K, Garcia MA, Ardissino D, Buszman P, Camici PG, Crea F, et al. Guidelines on the management of stable angina pectoris: Executive summary: The Task Force on the Management of Stable Angina Pectoris of the European Society of Cardiology. Eur Heart J 2006; 27: $1341-1381$.

25. Akabame S, Hamaguchi M, Tomiyasu K, Tanaka M, KobayashiTakenaka Y, Nakano K, et al. Evaluation of vulnerable coronary plaques and non-alcoholic fatty liver disease (NAFLD) by 64-detector multislice computed tomography (MSCT). Circ J 2008; 72: 618 625.

26. Hara T, Yamada S, Hayashi T, Ikeda Y, Yamashiro K, Mizutani K, et al. Accuracy of nonstenotic coronary atherosclerosis assessment by multi-detector computed tomography. Circ J 2007; 71: 911-914.

27. Motoyama S, Kondo T, Anno H, Sugiura A, Ito Y, Mori K, et al. Atherosclerotic plaque characterization by $0.5-\mathrm{mm}$-slice multislice 
computed tomographic imaging. Circ J 2007; 71: 363-366.

28. Becker A, Leber A, Becker C, Knez A. Predictive value of coronary calcifications for future cardiac events in asymptomatic individuals. Am Heart J 2008; 155: 154-160.

29. Dragano N, Verde PE, Moebus S, Stang A, Schmermund A Roggenbuck U, et al. Subclinical coronary atherosclerosis is more pronounced in men and women with lower socio-economic status: Associations in a population-based study: Coronary atherosclerosis and social status. Eur J Cardiovasc Prev Rehabil 2007; 14: 568-574.

30. Matsumoto N, Sato Y, Yoda S, Nakano Y, Kunimasa T, Matsuo S, et al. Prognostic value of non-obstructive CT low-dense coronary artery plaques detected by multislice computed tomography. Circ J 2007; 71: $1898-1903$

31. Cordeiro MA, Miller JM, Schmidt A, Lardo AC, Rosen BD, Bush $\mathrm{DE}$, et al. Non-invasive half millimetre 32 detector row computed tomography angiography accurately excludes significant stenoses in patients with advanced coronary artery disease and high calcium scores. Heart 2006; 92: 589-597.

32. Cademartiri F, Runza G, Mollet NR, Luccichenti G, Belgrano M, Bartolotta TV, et al. Impact of intravascular enhancement, heart rate, and calcium score on diagnostic accuracy in multislice computed tomography coronary angiography. Radiol Med (Torino) 2005; 110: $42-51$.

33. Giesler T, Baum U, Ropers D, Ulzheimer S, Wenkel E, Mennicke M, et al. Noninvasive visualization of coronary arteries using contrastenhanced multidetector CT: Influence of heart rate on image quality and stenosis detection. Am J Roentgenol 2002; 179: 911-916.

34. Herzog C, Arning-Erb M, Zangos S, Eichler K, Hammerstingl R, Dogan S, et al. Multi-detector row CT coronary angiography: Influence of reconstruction technique and heart rate on image quality. Radiology 2006; 238: 75-86.

35. Ehara M, Surmely JF, Kawai M, Katoh O, Matsubara T, Terashima
M, et al. Diagnostic accuracy of 64-slice computed tomography for detecting angiographically significant coronary artery stenosis in an unselected consecutive patient population: Comparison with conventional invasive angiography. Circ J 2006; 70: 564-571.

36. Leber AW, Knez A, von Ziegler F, Becker A, Nikolaou K, Paul S, et al. Quantification of obstructive and nonobstructive coronary lesions by 64-slice computed tomography: A comparative study with quantitative coronary angiography and intravascular ultrasound. $J$ Am Coll Cardiol 2005; 46: $147-154$.

37. Mollet NR, Cademartiri F, van Mieghem CA, Runza G, McFadden EP, Baks T, et al. High-resolution spiral computed tomography coronary angiography in patients referred for diagnostic conventional coronary angiography. Circulation 2005; 112: 2318-2323.

38. Pugliese F, Mollet NR, Runza G, van Mieghem C, Meijboom WB, Malagutti P, et al. Diagnostic accuracy of non-invasive 64-slice CT coronary angiography in patients with stable angina pectoris. Eur Radiol 2006; 16: 575-582.

39. Flohr T, Ohnesorge B. Heart rate adaptive optimization of spatial and temporal resolution for electrocardiogram-gated multislice spiral CT of the heart. J Comput Assist Tomogr 2001; 25: 907-923.

40. Flohr TG, Schaller S, Stierstorfer K, Bruder H, Ohnesorge BM, Schoepf UJ. Multi-detector row CT systems and image-reconstruction techniques. Radiology 2005; 235: 756-773.

41. Ropers U, Ropers D, Pflederer T, Anders K, Kuettner A, Stilianakis NI, et al. Influence of heart rate on the diagnostic accuracy of dualsource computed tomography coronary angiography. J Am Coll Cardiol 2007; 50: 2393-2398.

42. Husmann L, Schepis T, Scheffel H, Gaemperli O, Leschka S, Valenta $\mathrm{I}$, et al. Comparison of diagnostic accuracy of 64-slice computed tomography coronary angiography in patients with low, intermediate, and high cardiovascular risk. Acad Radiol 2008; 15: 452-461. 